\title{
Estilo directo e indirecto en interacciones orales: Estado de la cuestión en el ámbito hispánico*
}

\author{
María José Gallucci** \\ Universidad Central de Venezuela
}

INTRODUCCIÓN

Tradicionalmente se considera que la posibilidad de citar un discurso es un universal del lenguaje (Coulmas 1986) y que el estilo directo (ED) y el estilo indirecto (EI), como mecanismos de citación explícita que son, suelen definirse, respectivamente, como la reproducción literal de un decir o pensar ajenos; o bien como la reproducción de un dicho o un pensamiento en una oración subordinada que funciona como complemento del verbo de la oración principal (Lázaro Carreter 1990).

Sin embargo, la compresión del funcionamiento real y del alcance de estos dos procedimientos constituye uno de los grandes temas pendientes de la gramática del español. Esto no se debe, desde luego, a una falta de interés sobre el tema, pues vemos que en mayor o menor medida ha estado presente de alguna forma en las principales gramáticas del español, especialmente en las últimas ediciones. El artículo de Maldonado (1999)

* $\quad$ Esta investigación forma parte del proyecto $\mathrm{N}^{\circ}$ 07.8285-2011/1, titulado Las citas conversacionales en estilo directo e indirecto en el habla de Caracas, que cuenta con el financiamiento del Consejo de Desarrollo Científico y Humanístico de la Universidad Central de Venezuela.

** Para correspondencia dirigirse a: María José Gallucci (maria_gallucci@yahoo.es), Escuela de Letras, Facultad de Humanidades y Educación, Universidad Central de Venezuela, Caracas 1051, Venezuela. 
en la Gramática descriptiva de la lengua española (en adelante, GDLE) dedicado exclusivamente al discurso directo e indirecto y el amplio apartado (43.9) que se le dedica en la Nueva gramática de la lengua española (2010) (en adelante, NGLE) así lo demuestran; pero si cotejamos las definiciones y explicaciones que se ofrecen en los textos con el uso de estos tipos de citas en la interacción, observaremos que se trata de fenómenos complejos cuyo empleo trasciende muchas de las explicaciones que se ofrecen en las gramáticas del español. Esta situación puede deberse a que, tal como explica Maldonado (1999: 3554), "la cita es un fenómeno discursivo, no oracional"; o bien al uso de ejemplos que no proceden de interacciones reales (Camargo 2008b: 81).

Por todo lo antes expuesto, mi propósito es ofrecer aquí un estado de la cuestión lo más completo posible sobre el tratamiento del estilo directo e indirecto en el ámbito hispánico, con especial referencia a su funcionamiento en muestras orales, tanto en las gramáticas del español como en investigaciones que se han desarrollado recientemente en el contexto de la interacción conversacional. También, discutir el tratamiento de tales procedimientos, lo cual permitirá superar ciertos postulados teóricos que se dan por sentado, además de ofrecer una nueva perspectiva para su análisis.

\section{DEFINICIONES EN LAS GRAMÁTICAS DEL ESPAÑOL}

\subsection{GRamÁticas de la RAE 1973-1999}

Las gramáticas del español suelen incluir las cláusulas en estilo directo e indirecto en el grupo de las oraciones subordinadas sustantivas con función de objeto directo. Según el Esbozo de una nueva gramática de la lengua española de la Real Academia Española (RAE) de 1973, tanto las cláusulas reportadas en ED como en EI forman parte de las oraciones complementarias directas, específicamente de las oraciones enunciativas. Por tanto, se incluyen en el apartado de la subordinación sustantiva (3.19). En este apartado, los procedimientos de cita objeto de estudio se definen de la siguiente manera: "Llámese directo el estilo cuando el que habla o escribe reproduce textualmente las palabras con que se ha expresado el autor de ellas"; "En el estilo indirecto, el narrador refiere por sí mismo lo que otro ha dicho" (p. 516). Se aclara, igualmente, que en el caso del ED la subordinante y la subordinada están yuxtapuestas; mientras que en el EI se unen mediante la conjunción que y se altera el tiempo y modo de la cláusula subordinada. 
Gili Gaya 1998, al describir las oraciones complementarias directas, señala prácticamente lo mismo que se expresa en el Esbozo de $1973^{1}$. La novedad está en que el autor explica qué sucede cuando las oraciones subordinadas son varias y destaca el uso del que subordinante en el lenguaje popular y en la literatura:

Cuando las oraciones subordinadas son varias, puede llevar cada una la conjunción que, cosa muy frecuente en el lenguaje popular y en los clásicos: Decía el vizcaíno en sus mal trabadas razones que si no le dejaban acabar su batalla, que él mismo habia de matar a su ama (Quijote, I, 8). Hoy se tiende en el lenguaje literario a emplear la conjunción sólo con la primera subordinada, a no ser que la longitud del período pueda hacer olvidar la subordinación (Gili Gaya 1998: 288).

Seco (1998: 228), aunque no se refiere específicamente al estilo directo e indirecto, define la llamada yuxtaposición narrador-actor con ejemplos de tales procedimientos de cita:

Se trata de oraciones intercaladas en las que el narrador, al exponer en una oración las palabras textuales dichas por otro (o a veces sus pensamientos), añade una segunda oración que sirve de presentación de aquella: Las cosas -dijo (o pensó) Juan-son buenas o malas según el momento en que vienen; Dijo (o pensó Juan): -Las cosas son buenas o malas según el momento en que vienen; Las cosas son buenas o malas según el momento en que vienen -dijo (o pensó) Juan. Lo "dicho" o lo "pensado" por Juan es la oración Las cosas son buenas o malas según el momento en que vienen...

Alarcos Llorach (1999: 325), en el capítulo dedicado a las oraciones transpuestas sustantivas de su Gramática de la lengua española, señala, una vez más, aunque no le otorga mucha importancia al estilo directo e indirecto, la distinción entre oraciones yuxtapuestas y oraciones complejas:

En realidad, deben excluirse de las oraciones complejas las construcciones en que una oración en estilo indirecto se combina con otra (u otras) que reproduce el estilo directo y que, de estar transpuesta, funcionaría como objeto directo del núcleo verbal de la primera oración: Y me decía: "Te lo prometí, [...] te prometí que tu marido haría algo grande...". Mire, [el río] viene crecido -dijo, apagando el farol. Se trata de grupos de 
oraciones yuxtapuestas. Sólo serían oraciones complejas si se introdujese un transpositor: Y me decía que me lo había prometido..., Dijo que venía crecido.

\subsection{GRAmÁticAs DE LA RAE 1999-2011}

\subsubsection{Gramática descriptiva de la lengua española}

Una de las referencias principales sobre el tema que nos ocupa es el capítulo de Maldonado publicado en la Gramática descriptiva de la lengua española y que está dedicado exclusivamente a la explicación del funcionamiento del estilo directo e indirecto en español. Como se apreciará en lo sucesivo, se trata de un material que amplía el reducido panorama que ofrecen las gramáticas del español antes descritas sobre ambos procedimientos.

A partir de la definición del discurso directo e indirecto (1999: 3551) como la reproducción literal ${ }^{2}$ de palabras propias o ajenas, y la reproducción de esas palabras desde el sistema de referencias deícticas del hablante que reproduce (tiempo de la subordinada, pronombres, ciertos adverbios, etc.), respectivamente, Maldonado profundiza en la descripción sintáctica de las cláusulas en estilo directo e indirecto e identifica sus partes.

La autora explica que en su estructura canónica el ED suele estar constituido por una expresión introductora (EI) que contiene un verbo de reporte [generalmente conjugado], una cita directa (CD), marcada tipográficamente por guiones o comillas, y el contenido citado (CC), que siempre reproduce un enunciado. La EI y la CD están separadas por una pausa, que se marca tipográficamente con dos puntos (:).

Por su parte, el EI suele estar constituido por una expresión introductora (El) que contiene un verbo de reporte conjugado, una cita indirecta (CI), cuya marca suele ser la conjunción que, y el contenido citado (CC). La CI está subordinada al verbo de la EI.

La descripción sintáctica de ambos mecanismos se ilustra, respectivamente, en el siguiente ejemplo:

2 A pesar de que Maldonado le atribuye carácter literal al discurso directo, luego aclara (1991: 17) que "No importa que en la cita se reproduzcan palabras no emitidas realmente. Todo discurso citado, sea real o imaginario, supone necesariamente la reconstrucción de su situación de enunciación correspondiente". Más adelante (p. 20) acota que la cita directa puede no reproducir las palabras realmente emitidas. 
(1) a. [DD [EI Me dice: [CD “[CC Mira, te quiere conocer aquella chama que está allá]”]] (CARA_H11_001) ${ }^{3}$

b. [[DI [EI Yo simplemente le dije] [CI que [CC yo no quería estudiar más] ]] (CARA_H11_003)

A propósito de la sintaxis del estilo directo, la autora (p. 3565) explica que han sido muchas las soluciones propuestas para establecer qué tipo de relación hay entre la expresión introductora y la cita directa. Por esta razón, aunque se centra en la teoría que define el ED como una yuxtaposición de la EI y la $\mathrm{CD}$, explica las otras hipótesis en este sentido: i) el análisis de la cita directa como aposición de un deíctico, ii) la cita directa como un uso metalingüístico del lenguaje y iii) como complemento directo del verbo de decir ${ }^{4}$.

En el primer caso, en la EI se sobreentiende la existencia de un deíctico anafórico de la CD como en (2) y (3):

(2) Ella no dice nada a menos que tú le preguntes (esto): "mira, ¿qué tal?" (CARA_H13_075)

(3) Vamos a poner que me van a venir a reclamar (así): "mire, M." (CARA_H33_100)

En el segundo caso, es decir, al considerar la CD como uso metalingüístico, se asume que una oración que incluya un enunciado en una cita directa no reproduce el significado de dicho enunciado, sino que solamente lo nombra. De esta forma, la expresión citada se convierte en un "nombre" que significa la expresión en cuestión (p. 3556).

En el tercer caso, se trata de una postura que ha sido defendida entre varios de los gramáticos que han hecho referencia a la cita directa, tal como se puede apreciar en la primera parte de este apartado: la CD se considera objeto directo del verbo de decir de la EI. Por esta razón, el DD suele incluirse, como se ha visto, en los capítulos que tratan la subordinación sustantiva.

\footnotetext{
Los ejemplos presentados en $\S 1, \S 2$ y $\S 4$, al menos que se indique lo contrario, fueron extraídos del Corpus sociolingüistico del habla de Caracas, PRESEEA-Caracas 2004-2010 (cf. Bentivoglio y Malaver 2006). Cada conversación trascrita del corpus está identificada con un código alfanumérico que debe leerse de izquierda a derecha de la siguiente forma: i) CARA $=$ Caracas; ii) sexo: $\mathrm{h}=$ hombre, $\mathrm{m}=$ mujer; iii) grupo generacional: $1=$ de 20 a 34 años, 2 = de 35 a 54 años, 3 = de 55 años en adelante; iv) grado de instrucción del hablante: 1 = sin instrucción, 3 = más de quince años de escolaridad, universitario; y v) ubicación del hablante en la casilla del corpus.

4 Para más información sobre las críticas y los problemas que presentan cada una de las hipótesis, ver Maldonado (1999: 3565-3571).
} 
Sobre la sintaxis del estilo indirecto, la postura de Maldonado es similar a la que se asume en gramáticas anteriores: la CI funciona como complemento directo del verbo de decir y pertenecería al grupo de las subordinadas sustantivas. No obstante, la autora amplía la información que se ofrece en dichos textos y describe en detalle el comportamiento de la conjunción que (p. 3575) y acota que, en ocasiones, la CI no está constituida por oraciones propiamente, sino por fragmentos oracionales, que son enunciados incompletos pero que pueden ser interpretados contextualmente. Por ejemplo:

(4) a. Yo le dije: "bueno" (CARA_H11_002)

b. Nadie dice que no (CARA_H33_099)

Otro de los aportes de Maldonado (1999: 3555-3557) tiene que ver con las tres condiciones que deben cumplir los enunciados -en cualquier lenguapara ser considerados como citas: i) que sean la reproducción de una situación de enunciación; ii) que en la cadena verbal esté representado de algún modo el objeto del discurso (la materia verbal, no solamente la mención de un acto lingüístico); y iii) que la cita vaya introducida por verbos de decir en forma descriptiva, no realizativa ${ }^{5}$. Sobre este último aspecto llama la atención que, aunque Maldonado le dedica un breve apartado (p. 3558) a lo que denomina "cita de pensamientos" y explica la contraposición entre verbos de decir y verbos epistémicos, las citas solamente son consideradas como tales cuando están introducidas por un verbo de comunicación flexionado (p. 3565).

A la par del ED y el EI, Maldonado (p. 3552) también hace referencia a otros procedimientos de cita muy cercanos a estos. Se trata del discurso directo libre, el discurso pseudo-directo (o cita mixta) y el discurso indirecto libre.

Según Maldonado (1999: 3552), el discurso directo libre o "discurso directo sin marco explícito" reproduce los enunciados de forma literal; sin embargo, carece de un verbo que introduzca la cita. En estos casos, la cita puede conservar las marcas tipográficas, es decir, las comillas o los guiones ("discurso directo no regido"); o puede presentarse sin marca formal, a través de la entonación, lo que sería propiamente discurso directo libre.

\footnotetext{
5 Los verbos tienen valor realizativo cuando están en presente, en primera persona y cuando no suponen la descripción de una acción sino su realización (cf. Maldonado 1991: 28). Por ejemplo: "Yo puedo decir que [...] desde el noventa hasta el dos mil conocí [...] a los creadores de casi todos los libros de inglés" (CARA_H13_074).
} 
La cita mixta, también denominada "discurso pseudo-directo", se caracteriza por ser en su conjunto una cita indirecta con un verbo de decir y una oración subordinada encabezada por que, que contiene en su interior fragmentos de estilo directo; es, como su nombre indica, una forma de citación híbrida. En el siguiente fragmento, extraído de la prensa venezolana, se ilustra la cita mixta:

(5) Hugo Chávez, presidente de la República, le dijo a los partidos Patria Para Todos (PPT) y Partido Comunista de Venezuela (PCV) QUE VAN A "DESAPARECER" Del MaPa Político del país, a MENOS QUe SE UnAN AL Partido Socialista Unido de Venezuela (PSUV) [...]. Señaló Que el CANDIDATO APOYADO POR EL PPT Y EL PCV ES El CANDIDATO DEL "DIVISIONISMO" y reiteró QUE TANTO EL PCV COMO EL PPT DESAPARECERÁN DEL MAPA POLítICO DEL PAís (El Universal, edición online, 11 de octubre del 2008$)^{6}$.

Maldonado (1999: 3551) explica que el estilo indirecto libre se ha definido como un procedimiento a medio camino entre el ED y el EI y que esto ha justificado su inclusión en algunos estudios gramaticales. Esta técnica narrativa es propia del lenguaje literario y "consiste en la descripción de los contenidos de una conciencia de manera que el punto de vista del narrador y el punto de vista del personaje confluyan". Este mecanismo, como afirma la autora, supone siempre una ambigüedad comunicativa, ya que el receptor no puede saber con seguridad si lo que el narrador dice es responsabilidad suya o si se trata de un monólogo interior del personaje. Véase, a manera de ejemplo, el siguiente fragmento tomado de Maldonado (1999: 3552):

(6) Nerviosa se dijo: “¿Dónde habré puesto la cartera?” La había dejado allí encima, estaba segura. Quizá él la había descubierto y había leído lo que allí estaba escrito. ¡Con lo comprometedoras que eran aquellas palabras...! ¿Qué iba a hacer ahora?

\subsubsection{Nueva gramática de la lengua española}

En el segundo tomo de la Nueva gramática de la lengua española de 2009, centrado en el nivel de análisis sintáctico, se dedica un par de apartados más o menos extensos a la descripción de las características fundamentales del ED y el EI (\$43.9) y a las alternancias deícticas y mecanismos de traslación que tienen lugar en ambos mecanismos de citación (\$43.10).

6 Las versales son mías. 
El apartado $\$ 43.9$ comienza con la definición de estilo directo: "Se denomina discurso directo (DD) el que reproduce palabras pronunciadas de forma literal, así como los pensamientos transcritos de la misma forma en que se originan" (p. 3273). Como era de esperarse, el ED se ilustra con ejemplos extraídos de fuentes escritas.

Se explica (p. 3274) que por lo general el ED requiere la presencia de un verbo introductor, como decir, explicar, manifestar, opinar, repetir o responder, entre otros, y que la secuencia reproducida puede seguir al verbo introductor, como en (7), pero también puede precederlo, como en (8) o puede aparecer en posición medial, como en (9):

(7) Hubo gente que dijo: "bueno, yo no tengo pa' dónde agarrar" (CARA_ H13_074)

(8) “Ay, Doctor" me dijo ella (CARA_H33_097)

(9) Ella sacaba las morocotas, contaba mi abuela, y se las daba a los esclavos negros (CARA_M33_105)

Cuando la cita precede al verbo introductor, como en (8), el verbo se antepone al sujeto: me dijo ella... A propósito de este orden de palabras VS se señala que algunos gramáticos consideran que esta inversión es similar a un proceso de focalización, interpretación que le otorga al contenido de la cita una interpretación remática similar a la de los segmentos focalizados. Se especifica, además, que el DD puede introducirse como aposición al pronombre demostrativo esto, al adverbio demostrativo de modo así o a grupos nominales de interpretación deíctica: Dice esto, dice así, dijo lo siguiente.

Por su parte, el estilo indirecto se define de la siguiente manera:

el EI se caracteriza por presentar la voz del narrador. En este discurso se reproducen las palabras pronunciadas adaptándolas al sistema de referencias deícticas del hablante. Así, el fragmento Elsa dijo: "Mi hermana es famosa", que reproduce el discurso directo, corresponde a Elsa dijo que su hermana era famosa, que presenta esa misma información en el discurso indirecto (p. 3274).

En esta misma parte se destaca que el EI está sujeto a cierta indeterminación, pues sin contar algunas excepciones que se explican en el apartado

el oyente o el lector no posee instrumentos lingüísticos suficientes para dilucidar si un determinado texto introducido por un predicado que admite discurso directo representa o no una traslación de este. De hecho, en muchos casos no se da tal traslación (p. 3274). 
En el sub apartado 43.9d se explica que tanto ED como EI pueden ser libres y se afirma que una y otra variante es propia del texto literario. Como especificaba Maldonado (1999), el ED libre "se caracteriza por la ausencia de verbos de lengua o de pensamiento, así como de otras marcas lingüísticas que separen el texto que corresponde al narrador del que pertenece a los personajes" (p. 3275); mientras que el EI libre "se caracteriza por alternar los centros deícticos que corresponden al narrador y a los personajes del texto narrado". Los ejemplos que se emplean para explicar ambos procedimientos de citas provienen de textos literarios.

Luego, se le dedican varios sub apartados (pp. 3276-3279) a las interrogativas indirectas y su relación con el ED. Si nos remitimos al funcionamiento de estas en textos orales, en la NGLE destacan varios aspectos. En primer lugar, la alusión a la estructura "que + interrogativa indirecta", característica del registro conversacional, como en (10). En segundo lugar, los ejemplos que se ofrecen del uso del verbo decir con el significado de "preguntar" (11).

(10) Me pregunta que qué quiero estudiar (CARA_M33_105)

(11) Entonces yo digo: ¿qué pasó? (CARA_H13_073)

Otro aporte que ya había hecho Maldonado (1999) y que se rescata en la NGLE (p. 3279) tiene que ver con el hecho de que el EI puede introducir fragmentos sintácticos menores que la oración, lo que se explica de la siguiente manera:

Esta posibilidad permite diversos tipos de elipsis en el discurso reproducido, si bien sometidos a ciertas restricciones. En efecto, la secuencia Ella respondió que a las cuatro podría usarse en varios contextos. Por un lado, podría dar fin a un período si en el discurso previo se proporciona la información verbal que en ella se omite, como en $L e$ preguntó a qué hora deseaba salir, y ella respondió que a las cuatro. Por otro lado, sería también posible que la oración propuesta trasladara el DI a un determinado DD: Ella respondió: "A las cuatro". Esta opción es particularmente frecuente cuando el DI reproduce manifestaciones de conformidad o disconformidad en relación con la información aportada en el texto precedente $[. .$.$] Desde el punto de vista de su estructura interna$ no son oraciones, pero sí enunciados, ya que presentan un discurso reproducido integrado en la oración principal. Admiten, además, la sustitución por pronombres átonos, como en Dice que adelante y lo dice a conciencia. 
Aunque no es habitual trasladar al DI las interjecciones, imprecaciones y otras expresiones exclamativas, en el texto se admite que "se registran algunas excepciones en la lengua oral relajada". La excepción, según la NGLE, la constituye un caso en el que el EI puede contener, ocasionalmente, interjecciones: Y ya uno decía que ;coño!, nunca uno, nunca, no puede pensar en pajaritos y bromas así (p. 3280). Este ejemplo pertenece al CREA oral, Venezuela.

\subsubsection{Nueva gramática de la lengua española. Manual}

En el Manual de la RAE (2010), como se sabe, se presentan los contenidos de la NGLE pero de forma abreviada.

La primera referencia al ED y el EI se ofrece en $\S 43.1$ (p. 829), en el apartado sobre interrogativas y exclamativas indirectas. Las interrogativas indirectas son las subordinadas sustantivas encabezadas por los pronombres, determinantes o adverbios interrogativos (o por los grupos sintácticos que forman), o bien por la conjunción subordinante si: Pregúntale si quiere venir con nosotros, que equivale formalmente a una interrogativa directa, como muestra su equivalente en estilo directo Pregúntale: ¿Quieres venir con nosotros? ${ }^{7}$

El apartado 43.4 (p. 833) de la obra es el que está dedicado al ED y EI. En él se señala, una vez más, que el ED reproduce de forma literal palabras o pensamientos. También se hace alusión al hecho de que suele aparecer con un verbo introductor que ocupa diversas posiciones. En cuanto al EI, la definición apunta nuevamente a la reproducción de las palabras de otro, adaptándolas al sistema de referencias deícticas del hablante. Se ofrecen ejemplos de ambos procedimientos de cita y se destaca que en ambos tipos de discurso es posible introducir fragmentos sintácticos menores que la oración. También se hace alusión a las interrogativas indirectas propias que trasladan el discurso directo:

Su característica sintáctica más notable es que están introducidas por la conjunción que. Así, la oración Le preguntó que cuándo llegaba el tren se corresponde con Le preguntó: “¿Cuándo llega el tren?”. En estos casos la conjunción que es potestativa con preguntar, pero es forzosa con los demás verbos de lengua que introducen el estilo directo (decir, gritar, chillar, susurrar) si se quiere indicar que lo que se transmite es

\footnotetext{
7 En el apartado, el término "interrogativa indirecta" no se toma en sentido literal, puesto que solo algunas de estas oraciones reproducen el discurso directo.
} 
una pregunta. En efecto, en la oración Le dijo cuántos habían asistido al concierto se dice que alguien comunicó a otra persona el número de asistentes, mientras que en Le dijo que cuántos habian asistido al concierto se dice que alguien formuló cierta pregunta. Se rechaza la conjunción con los verbos que no introducen el discurso directo, aunque sean verbos de lengua, como en Elsa nos contó \{por qué *que por qué\} había fracasado el proyecto. La variante con que es compartida también por las interrogativas totales (Le preguntó que si hacia frío), por las interrogativas que dependen de ciertos sustantivos (No hacía más que repetir la pregunta de que cuándo le tocaba a él) y por las exclamativas indirectas, como se vio en el apartado precedente: No es imposible que él replicara que qué preparación tenía ella para sustituir a la Castell (Luca Tena, Renglones) (p. 834).

La sección dedicada al ED y el EI finaliza con la descripción de las relaciones entre ambos mecanismos de citación. Tal y como se expresa en la NGLE, se destaca la indeterminación a la que está sujeto el EI. Esta indeterminación se debe, por una parte, a que el receptor no siempre es capaz de reconstruir a través de él las palabras pronunciadas en el ED correspondiente. Por otra parte, obedece a que mientras que en el ED el discurso se estructura en torno al narrador, es decir, al emisor de las palabras que se trasmiten, en el EI se estructura en torno al hablante. Esto último, como se ha visto, da lugar a cambios en los centros deícticos si el narrador y el hablante no comparten el mismo espacio y el mismo tiempo.

\subsubsection{Nueva gramática básica de la lengua española}

Tal y como se expresa en las primeras páginas de la obra (2011: xvii), en este caso se trata de una versión que "se dirige expresamente al amplio espectro de hispanohablantes que, habiendo recibido una primera instrucción en sus estudios de primaria y de secundaria, deseen acercarse a comprender mejor el funcionamiento de su lengua". En virtud de su carácter general, las referencias en el texto sobre el ED y el EI se limitan a las definiciones que se ofrecen en el capítulo 25 dedicado a las oraciones subordinadas sustantivas (p. 41). Aunque se repite parcialmente y de forma muy breve lo que hemos visto en los dos apartados anteriores, llama la atención que el ED, a pesar de que sigue siendo definido a partir de la literalidad, de la posición de cláusula de reporte y prácticamente con los mismos ejemplos, se acota que las palabras citadas o los pensamientos pueden ser "propios o de otra persona" (p. 241). 


\section{ANÁLISIS DEL ESTILO DIRECTO E INDIRECTO EN INTERACCIONES ORALES}

En los últimos años, los estudios sobre las distintas formas en que puede citarse en español se han centrado, fundamentalmente, en el análisis de textos escritos del discurso académico, periodístico o literario. Quizás esto se deba a la dificultad que supone encontrar corpus orales idóneos $y$, aunado a esto, al hecho de que el análisis de las conversaciones suele ser más complejo, entre otras cosas, en virtud del alto grado de indefinición, imprevisibilidad e improvisación por parte de quienes intervienen en ellas (cf. Kerbrat-Orecchioni 1996) ${ }^{8}$. Esto ha dificultado la comprensión cabal del funcionamiento del ED y el EI en la oralidad, modo de manifestación del discurso por excelencia.

En este apartado presento algunas investigaciones importantes que se han hecho en los últimos años, específicamente sobre el uso del ED y el EI en español, utilizando como corpus de estudio muestras provenientes de interacciones orales. La idea es mostrar, como se ha dicho, el panorama en torno al análisis de ambos mecanismos de citación explícita en el ámbito hispánico.

En primer lugar, describo los aportes de varios estudios pioneros sobre el español de América (Cameron 1998; van der Houwen 1998; Mora y Álvarez 2003; Guirado y Shiro 2004; Mateus 2005; Gallucci 2009; García 2009; Fernández 2011, San Martín y Guerrero 2012).

En segundo lugar, hago referencia a un algunos estudios que se han hecho sobre los procedimientos de cita en el español de España (Benavent Payá 2003, Camargo 2004a).

8 Sobre este aspecto, Thompson (1994: 164) explica que "es más difícil delinear el contexto del reporte en la conversación que en el periodismo, la literatura, o en los artículos académicos, ya que hay menos reglas obvias a propósito de lo que se dice o de las distintas formas en las que se puede decir algo. No obstante, aun en la conversación, tanto el hablante como el oyente comparten ciertas expectativas sobre los tipos de reporte que tendrán lugar" ['It is more difficult to outline the context of reporting in conversation than in journalism, novels, or academic articles, because there are fewer obvious rules concerning what can be talked about or the ways in which you can talk. However, even in conversation the speaker and listener do share certain expectations about the kinds of reporting that will occur', MT]. 


\subsection{Estudios EN EL ESPAÑOl de AmÉRICA}

\subsubsection{Cameron 1998}

Cameron 1998 analiza, desde una perspectiva variacionista, las formas del discurso directo en una muestra de habla de 62 hablantes nativos de San Juan, Puerto Rico. El autor distingue tres usos variables para introducir el discurso directo en la conversación: i) mediante un verbo de decir (Entonces yo DIGO, “'Ahora prepárate, que te voy a quitar un montón de cosas!”); ii) $y+$ frase nominal (Y ELLA, “;Ah no, mijo!"; y iii) sin marco o freestanding quotation (Entonces me metía escapado y qué sé yo para la tienda Ø “¿Qué pasó?" "No tengo clase"). En este estudio el autor encuentra un total de 1249 casos, de los cuales 774 (62\%) se introducen mediante un verbo de decir, $310(25 \%)$ sin marca y $165(13 \%)$ mediante la conjunción $y$ seguida de una frase nominal. Los tipos de verbos encontrados y su frecuencia en el corpus estudiado son: decir (643 casos, 83\%); hacer (50 casos, 6\%); verbos de movimiento (22 casos, $2 \%) ;{ }^{9}$ gritar (9 casos, $1 \%$ ); preguntar y cuestionar ( 8 casos, $1 \%)$; pensar ( 8 casos, $1 \%)$; venir + asi ( 7 casos, $1 \%$ ); y otros verbos (26 casos, $3 \%$ ): quedarse, avisar, predecir, contestar, llamar, empezar, responder.

Otros de los hallazgos de Cameron tienen que ver con la persona a quien se le atribuye el reporte, el contenido de la cita, el contexto narrativo y el tópico como variables lingüísticas que inciden en el uso de las tres formas del ED propuestas por el autor.

En cuanto al primer aspecto, Cameron (1998: 64) sostiene que cuando las citas se introducen mediante un verbo de decir o de $y+$ frase nominal, los hablantes privilegian el uso de la primera persona del singular ( $Y O$ dije), seguida de la tercera persona del singular (Él dijo). El autor también encuentra que la fuente de la cita suele ser un ente animado y que el rasgo inanimado se ve favorecido, sobre todo, por las citas sin marco.

En cuanto al segundo aspecto, el contenido de la cita, el autor establece una triple distinción: si se trata de una cita de palabras (Juan me dijo que yo era muy bonita); solamente de interjecciones, exclamaciones o risas (;Ay Dios mío!); o de un gesto acompañado de un sonido (porque hice así:

\footnotetext{
$9 \quad$ En este grupo el autor incluye los verbos coger, dar, echar, levantar, mirar, seguir, subir y tirar seguidos de así, en virtud de que su análisis contempla también las citas de gestos. Aunque las conversaciones analizadas por este autor no fueron filmadas, el autor infiere la presencia de algunos gestos tomando como patrón estructuras lingüísticas seguidas del adverbio así: Y ella lo miraba así [gesto] (p. 48).
} 
"[gesto] fuuuuuuum"). De esta variable el lingüista explica que el reporte de palabras se ve favorecido por el empleo de un verbo de decir; el uso de interjecciones, por la estructura $y+$ frase nominal; y el reporte sin marco introductor, por los gestos acompañados de sonidos.

El tercer hallazgo, la incidencia de los contextos narrativos (p. 67), muestra que los contextos narrativos favorecen el uso de $y+$ frase nominal, mientras que los contextos no narrativos favorecen la ausencia de marco introductor. Según el tópico de la cláusula, Cameron señala que el miedo, el humor o la sorpresa favorecen la presencia de $y+$ frase nominal, y que la categoría "otros" favorece la presencia de un verbo de decir para introducir la cláusula reportada.

El análisis de las tres variables extralingüísticas consideradas por el autor -edad (preadolescentes, adolescentes, de 20 a 39 años, y de más de 40 años); sexo (hombres y mujeres) y clase social (alta y baja)- dio como resultado que: i) los dos grupos de más edad favorecen la presencia de un verbo de reporte y desfavorecen tanto la presencia de $y+$ frase nominal como la cita sin marco; ii) la estructura $y+$ frase nominal y las citas directas $\sin$ marco se correlacionan con la edad de los hablantes, en este caso con la de los jóvenes; iii) cuando se trata de $y+$ frase nominal la clase social de los hablantes no influye en la variación, mientras que la presencia de un verbo para introducir la cita y la cita sin marco sí lo hacen, y están en distribución complementaria: el verbo es favorecido por la clase baja y desfavorecido por la clase alta, y sucede lo contrario con la cita directa sin marco; iv) la variable sexo no influye en la presencia de un verbo de reporte para introducir la cita directa, pero son las mujeres, y no los hombres, las que prefieren usar $y$ + frase nominal; el efecto contrario se observa con las citas directas sin marco introductor (cf. Cameron 1998: 70-71).

\subsection{2. van der Houwen 1998}

Van der Houwen 1998 analiza cualitativa y cuantitativamente el uso del ED y del EI en el habla de la Ciudad de México. Esta autora seleccionó como corpus de estudio 15 conversaciones transcritas, de treinta minutos de duración, de mujeres entre 20 y 80 años de edad, pertenecientes al nivel culto y al popular. Estas muestras, que suman en total siete horas y media de conversaciones, fueron extraídas de dos corpus publicados por el Centro de Lingüística Hispánica de la Universidad Nacional Autónoma de México (cf. Lope Blanch 1972 y 1976). La autora (1998: 125) plantea como hipótesis que cuando el hablante usa el EI introduce al oyente en un escenario comunicativo distinto, mientras que cuando usa el ED este último ya ha sido situado directamente en un escenario comunicativo específico en el que el hablante ha establecido su centro deíctico. La autora solamente 
toma en cuenta el verbo decir y, con base en esto, identifica tres formas de introducir el reporte en estilo directo o indirecto: a) ED acompañado del verbo decir; b) EI acompañado del verbo decir; y c) ED sin ningún verbo de decir. Los hallazgos generales de van der Houwen 1998 revelan que los hablantes: i) citan más en ED que en EI (507 casos vs. 90), lo que confirma, una vez más, que el reporte en estilo indirecto es la estructura marcada, y que el ED es la no marcada; ii) suelen introducir el reporte en ED con el verbo decir ( $64 \%$ de los casos vs. $21 \%$ sin ningún verbo de decir); iii) usan los verba dicendi en presente histórico y con el ED, más que con el EI (227 casos vs. 28 casos), como en En ese momento, estábamos conversando y el hombre le dice: "No saltes"; iv) emplean mucho más el tiempo pasado que el presente cuando reportan en EI (62 casos vs. 28 casos); v) suelen elidir el sujeto cuando reportan en ED (286 casos de ausencia del sujeto vs. 95 casos de presencia). A partir de estos resultados, van der Houwen (1998: 132) sostiene que el ED y el EI tienen un efecto comunicativo y funcional distinto y que, por lo tanto, su uso no es aleatorio, pues depende del mensaje que el hablante quiera transmitir.

\subsubsection{Mora y Álvarez 2003}

Mora y Álvarez 2003 estudian el discurso indirecto y sus características acústicas y prosódicas en el español de Venezuela. A partir del análisis de fragmentos de habla espontánea de doce voces femeninas de diferentes regiones del país, de las que se extrajeron 56 emisiones de cláusulas reportadas, las autoras encuentran diferencias en cuanto a tono, intensidad y duración. Los resultados contribuyen a esclarecer algunos aspectos relevantes sobre el uso de los procedimientos de cita (p. 573): i) "se comprueba que la representación del habla en el diálogo, como sostiene Tannen [...], es un acto narrativo"; y ii) las entradas del discurso subordinado (el discurso del otro) en el discurso dominante "no son copias fieles de lo que se dice, sino que si bien son icónicas, probablemente no son idénticas y por lo tanto sólo son verosímiles".

\subsubsection{Guirado y Shiro 2004}

Guirado y Shiro 2004 analizan las voces reportadas en una muestra de 419 narraciones de ficción y experiencia personal producidas por sesenta y ocho niños de primer y cuarto grado. Entre los resultados de este estudio sobre el habla infantil de Caracas destacan dos aspectos: i) una marcada tendencia de los niños a reportar la información en ED; ii) en las narraciones, los varones utilizan el habla reportada con mayor frecuencia que las mujeres. 


\subsubsection{Mateus 2005}

Mateus 2005 estudia cuantitativa y cualitativamente ambos mecanismos de cita en un corpus oral de 32 grabaciones de entrevistas, 27 tomadas del Corpus sociolingüístico de Caracas de 1987 (cf. Bentivoglio y Sedano 1993) y 5 realizadas por la autora en estilo informal y espontáneo a hombres caraqueños de nivel socioeconómico alto, medio y bajo. El objetivo principal de la autora es corroborar, en narraciones producidas por hablantes caraqueños, la hipótesis de van der Houwen 2000 (también esbozada en su artículo de 1998) en cuanto a que tanto el ED como el EI tienen una función comunicativa propia, que obedece a factores pragmático-discursivos. Los principales resultados del análisis de Mateus 2005, además de confirmar que decir es el verbum dicendi más utilizado, muestran que los hablantes: i) prefieren narrar más en ED que en EI ( $80 \%$ de los casos frente a $20 \%)$ y, en especial, en los momentos estelares del relato; ii) cuando narran utilizan más el presente histórico (es decir, emplean el tiempo verbal presente en la narración de hechos pasados), sobre todo con el ED y en los momentos de clímax del relato; y iii) eliden el verbum dicendi cuando narran en ED los pasajes dramáticos. Estos resultados confirman la hipótesis de van der Houwen 2000.

\subsubsection{Gallucci 2009}

Gallucci 2009 analiza una muestra de aproximadamente 4 horas y media de grabación (6 hablantes, 3 hombres y 3 mujeres, de 55 años o más, y de grado de instrucción universitario) del Corpus sociolingüístico de Caracas 2004-2008 (cf. Bentivoglio y Malaver 2006). En esta oportunidad, de los 487 casos encontrados se observa que: i) en $93 \%$ del total de los casos (453) los hablantes reportan en estilo directo, y solamente en un $7 \%$ lo hacen en estilo indirecto (34); ii) los hablantes, aunque pueden introducir la cláusula reportada en ED o EI al menos de cinco formas (mediante un verbo de reporte, $\sin$ verbo, con $(y)+\mathrm{SN},(y)+q u e$ y a través de un marcador discursivo) suelen introducir las citas, sobre todo, a través verbo conjugado $(37 \%, 178$ casos) o sin él $(34 \%, 168$ casos); iii) el verbo de reporte más empleado es decir (154 casos de 178); iv) los hablantes prefieren citarse a sí mismos $(56 \%, 273$ casos); v) cada cita se corresponde con una función discursiva específica; entre ellas, las más usadas fueron: relatar una anécdota (61\%) y reportar un pensamiento (19\%); y vi) los hombres jóvenes (de 20-34 años de edad) citan más que las mujeres: 367 casos (75\%) vs. 120 casos $(25 \%)$. 


\subsubsection{García 2009}

García 2009 estudia el discurso referido, desde un punto de vista esencialmente cualitativo, en 7 hablantes extraídos de dos fuentes principales: i) un corpus levantado en 1982, como parte de un proyecto que pretendió dar cuenta del español hablado en las vecindades de la ciudad de Puebla (Gimate-Welsh 1985) y ii) el corpus del español de Puebla a cargo del Dr. James L. Fidelholtz. El primer corpus está constituido por 3 hablantes: 2 mujeres (de 36 y 42 años) y 1 hombre (44 años). El segundo, por 2 hombres ( 22 y 33 años) y 2 mujeres ( 20 y 23 años). En este último corpus, el autor seleccionó los participantes que guardan relaciones sociales cercanas hermanos o amigos íntimos-, ya que esto provoca un contexto de confianza entre los hablantes que les permite hablar con comodidad. Aunque se describe la edad de los hablantes que forman parte del corpus, esta variable no se considera en el estudio.

La pregunta de investigación central que se hace el autor es: ¿existe relación entre las formas del discurso referido y las funciones pragmáticoargumentativas? García $(2009$, p. 17) afirma que sí existe relación entre estos dos aspectos, ya que los hablantes "cuando quieren lograr alguna reacción en sus interlocutores pueden hacerlo a través del uso del discurso referido como parte de una estrategia de más largo alcance".

Las funciones identificadas por el autor fueron clasificadas en dos grandes categorías: la presentación de la voz de otras personas y la argumentación. Se trata de dos clasificaciones que, como indica el mismo autor, encierran de manera general los resultados del análisis. En este sentido, García explica (pp. 117-118):

Encontramos que un hablante puede simplemente usar las palabras de otra persona o de sí mismo valiéndose principalmente del discurso directo, para alejarse simbólicamente de lo dicho y no sufrir las consecuencias que vengan tras las palabras. Es decir, el hablante en todo momento está monitoreando a su interlocutor y puede modificar su estrategia para que no se le escape de las manos, a este uso lo clasificamos dentro del discurso referido como crédito de autoría. [...] La argumentación a través del discurso referido es mucho más compleja que el crédito de autoría, pues éste sólo busca separarse de las consecuencias de las palabras, mientras que la argumentación busca lograr más cosas en el interlocutor y sus estructuras son más variadas.

En cuanto a la frecuencia de uso del discurso referido, el autor constató la diferencia en relación con el número y tipo de casos encontrados en los dos diferentes grupos que conformaron el corpus de la investigación: mientras 
los datos de 1980 arrojaron un número mucho mayor de discurso indirecto, los datos del año 2000 tuvieron un número más alto de discurso directo.

La explicación que le da el autor al comportamiento de los datos antes descrito es que el formato de entrevista "tradicional" (pregunta-respuesta) busca sondear aspectos específicos según la temática planteada por el entrevistador, lo que permite al hablante inaugurar cada vez un acto de reporte diferente prácticamente tras cada pregunta. Por otro lado, las repuestas al formato de interacción que se encuentra en los datos del 2000 regularmente se enfocan en temas propuestos por el entrevistador, pero desarrollados ampliamente por el informante. Es importante observar el desarrollo que el hablante hace de sus temas, pues en los datos disponibles del grupo del año 2000 el hablante tiene más libertad para continuar con un tema que le interesa: presentar detalladamente su objetivo conversacional, hipótesis o sentencia inicial, argumentar sobre esa sentencia inicial y volver a su hipótesis para corroborarla. Esto le permite asegurar a García que la metodología de levantamiento de datos en el caso del discurso referido condiciona el tipo de datos que se obtendrán.

En el análisis se observa también que el verbo más usado para introducir el discurso referido es el verbo de habla decir, tanto en discurso directo como en indirecto, seguido de otros verbos como contar, exigir, preguntar y aceptar, o inclusive acepciones especiales de verbos como salir. Los verbos de pensamiento creer y pensar son los que tuvieron, relativamente, más usos detrás del verbo decir.

El autor señala que los hablantes tienen la posibilidad de omitir el verbo introductorio, la mayoría de las veces cuando se enumeran actos de habla uno tras otro, y en casos menos evidentes como cuando presentan dos reportes de habla casi simultáneamente.

\subsubsection{Fernández 2011}

Fernández 2011 describe el uso del estilo directo e indirecto en 6 transcripciones del Corpus sociolingüistico de Mérida (Venezuela) 20092010 correspondientes a 3 hombres y 3 mujeres de distintos grupos generacionales.

La autora analiza la frecuencia con la que los hablantes usan el ED y el EI, los verbos introductores de cita, la presencia de la conjunción que, la preferencia por la autocita o la heterocita, el uso de los mecanismos de cita según la edad y el sexo de los hablantes y, por último, las funciones pragmáticas de la cita.

En total, Fernández encontró 80 casos de discurso reportado. De estos casos, 56 corresponden al ED y 24 al EI. 
Los resultados más importantes del análisis de estos casos muestran que: i) los hablantes prefieren usar el ED para reportar ( $70 \%$ de los casos) y que la mayoría de las veces presentan el reporte con un verbo de comunicación ( $71 \%$ del total de casos); ii) el verbo más empleado para reportar es decir tanto en el ED (92\%) como en el EI (90\% de los casos); iii) en relación con la presencia y la ausencia de la conjunción que, los hablantes al momento de reproducir en ED tienden a elidir la conjunción en un $88 \%$, mientras que cuando citan en EI se observa la tendencia opuesta: los hablantes emplean la conjunción en un $87 \%$ de los casos de EI encontrados en la muestra; iv) hay mayor empleo de la heterocita que de la autocita en hombres y mujeres; v) en cuanto a las funciones pragmáticas, la más empleada en ED es ejemplificar y en EI, relatar; vi) en relación con el sexo y la edad de los hablantes, tanto hombres como mujeres de los tres grupos generacionales emplean con mayor frecuencia el ED para reportar enunciados.

\subsubsection{San Martín y Guerrero 2012}

San Martín y Guerrero 2012 analizan el empleo del discurso referido en el habla de Santiago de Chile desde un punto de vista sociolingüístico. Para tal fin, toman como corpus de estudio 54 entrevistas que forman parte del Corpus Sociolingüístico del PRESEEA en Santiago de Chile.

Los investigadores consideraron el discurso referido como un caso de variable sociolingüística, en un sentido amplio del concepto, con dos valores o variantes generales: el discurso directo y el discurso indirecto y con diferentes subtipos o variantes específicas de acuerdo con distintos niveles o grados de reformulación y distanciamiento del sujeto respecto de su enunciado.

Entre los resultados obtenidos destacan: i) mayor uso de ED (74,2\%) en comparación al EI $(25,8 \%)$; ii) mayor uso de ED $(68,8 \%)$, EDL (estilo directo libre) $(28,9 \%)$ y escasa frecuencia de EDPRO (discurso directo introducido por frases nominales) $(2,3 \%)$; iii) en el EI: mayor uso de EI $(62,4 \%)$ en comparación al EIL (37,6\%); iv) el discurso referido aparece mayoritariamente en secuencias narrativas $(61,8 \%$ el ED y $19,2 \%$ el EI) en comparación a las argumentativas (13\% en el ED y $6 \%$ en el EI).

En cuanto a las variables sociales, San Martín y Guerrero observan una frecuencia similar según el sexo-género, pero mayor porcentaje en mujeres $(40,8 \%$ en ED y $14,6 \%$ en EI, frente al $33,5 \%$ ED en los hombres y $11,1 \%$ en EI). Esto último les hace sugerir que las mujeres suelen emplear con mayor frecuencia el discurso directo como una estrategia pragmática mediante la cual intentan dotar de mayor teatralidad a sus narraciones en forma de "mini-dramas". 
En relación con el grupo de edad, el ED se manifiesta con porcentajes similares en el primer y tercer grupo ( $26 \%$ y $27,1 \%$, respectivamente); mientras que en el segundo grupo la frecuencia de ED disminuye $(21,1 \%)$. El uso de DI, por su parte, disminuye conforme avanza la edad: 11\% (grupo I), 9,4 (grupo II) y 5,4 (grupo III).

Respecto del nivel educacional, en la muestra objeto de estudio el ED presenta mayor frecuencia en el nivel medio $(31,8 \%)$ y disminuye en los niveles primario $(23 \%)$ y alto $(19,5 \%)$. Por su parte, el EI se manifiesta de manera similar en los tres grupos: $8 \%$ (bajo), $7,7 \%$ (medio) y $10 \%$ (alto).

\subsection{Estudios EN EL ESPAÑOL DE EsPaÑa}

\subsubsection{Benavent Payá 2003}

Benavent Payá 2003 hace un estudio sobre el corpus del español coloquial publicado por el grupo Val.Es.Co (Briz 1995). El objetivo principal del estudio es analizar el uso de algunas estructuras sintácticas del discurso reproducido que podrían deberse a factores cognoscitivos.

La investigación de esta autora está motivada por la constatación de que en español (2003: 11) hay "un claro predominio del discurso directo frente al indirecto cuando contamos nuestras vivencias".

Para explicar las razones que favorecen su uso en los relatos conversacionales, Benavent Payá 2003 aplica la perspectiva cognitiva de Fauconnier 1984 sobre los espacios mentales y las ideas de Achard 1998 y Delbecque 2000 sobre el discurso reproducido.

La investigación se centra en tres aspectos: el significado del verbo decir, la estructura de la cita y la relación que se establece entre ambos. En las conclusiones destacan dos aspectos que contribuyen a la explicación del uso del ED y del EI en la interacción: i) cuando el hablante usa el DI (perspectiva unificada) solamente pretende comunicar a su interlocutor el contenido básico de la situación enunciativa reproducida; en cambio, cuando usa el ED (perspectiva desdoblada) persigue un objetivo añadido, relacionado con el papel del oyente que escucha la historia; y ii) "los mecanismos que estructuran estas historias parecen reflejar no tanto un modo particular de percibir el mundo, como sí una estrategia para implicar al oyente de la historia y persuadirle sobre la veracidad de la misma" (Benavent Payá 2003: 17). 


\subsubsection{Camargo 2004}

Camargo 2004a, una de las autoras que más ha profundizado en el estudio de la reflexividad lingüística en español ${ }^{10}$, analiza, desde la sociopragmática, diferentes formas de representación del discurso en la narración oral conversacional: cita directa lingüística (una señora de Madrid dice: ";madre mía yo me pongo un abrigo de piel, y al día siguiente me han robado!"); citas no lingüísticas: cita de paralenguaje (el plato se cayó pero no se cayó "plin") y de gestos (yo me tengo que ver así [gesto con la cabeza mirándose el cuerpo]); citas indirectas (y se acercó uno, que le diésemos dinero $)^{11}$; citas cuasi indirectas (según te dicen, traen aquí pero cargamentos); comentarios metadiscursivos (lo que te hablaba antes o te ponías en una tienda a trabajar o hacias cualquier cosa porque más no se podia hacer); narración de acción lingüística (sólo hablaba de él y el tatuaje) y narración de voz (no te dicen nada).

La autora hace su estudio en dos corpus: el ALeCMan-Cuenca (cf. García Mouton y Moreno Fernández 2003) conformado por entrevistas de formalidad máxima, media y mínima) y el Corpus Espontáneo (con entrevistas solo de formalidad mínima) recogido por la misma autora con grabadora oculta. El total de formas de representación del discurso analizadas por Camargo en los dos corpus fue de 821, de las cuales 512 pertenecen al ALeCMan-Cuenca y 309, al Corpus Espontáneo.

Del total de formas halladas por Camargo (2004a: 244), 745 son citas de lenguaje, paralenguaje y gestos (en este grupo se incluyen las citas directas, las no lingüísticas y las indirectas); y 76 son comentarios metadiscursivos. De los 512 casos del Atlas, 347 son citas directas lingüísticas, 9 son citas no lingüísticas, 99 son citas indirectas y 57 son comentarios metadiscursivos. En el Corpus Espontáneo, de los 309 casos hallados, 224 son citas directas; 57 demostraciones no lingüísticas; 9 son citas indirectas; y 19, comentarios metadiscursivos. Estos resultados le permiten afirmar a la autora que:

la cita conversacional es, primordialmente, directa, al menos en las secuencias narrativas orales [...]. Los datos con los que hemos trabajado

10 Aunque Camargo cuenta con muchos trabajos sobre el discurso reportado (2004b, 2005, 2008a, 2008b, 2008c, 2010, 2011) que constituyen, sin duda, un aporte fundamental para entender el uso de las citas, en el apartado describo los hallazgos generales producto de la tesis doctoral de la autora (2004a).

11 En este grupo, Camargo (2004a: 223-224) incluye también "las citas indirectas sin contenido proposicional" del tipo: alli estaban mis tíos en la casa y estaban comentando con mis padres pues que habian bombardeado que no sé qué que no sé cuantos. 
revelan que las citas directas representan un porcentaje muy alto del total de casos de las formas de representación del discurso que aparecen. (Camargo 2004a: 244)

Algunas de las conclusiones generales más importantes a las que llega Camargo (2004a: 250-253) son: i) las citas de paralenguaje del Corpus Espontáneo (respiración audible, risas, gritos, etc.) siempre aparecen en los relatos dramatizados acompañando a citas lingüísticas, mientras que las citas de elementos cuasiléxicos en el Atlas (сисисисиси́, pum ta ta pum pum pum, etc.) aparecen de forma aislada, pero no como parte de una representación dramatizada de enunciados; ii) en el ALecMan-Cuenca el comportamiento de las mujeres es muy similar en cuanto al uso de las citas, y los distintos modos de citar de estas no están relacionados con la edad ni presentan tendencia al cambio; iii) los datos extraídos del Corpus Espontáneo revelan que, en lugar del grado de instrucción, los factores que más influyen en los modos de citar son el auditorio y la situación comunicativa; iv) en el caso de los informantes de sexo masculino del ALecMan-Cuenca, el nivel de instrucción y la edad sí influyen en el uso que los hombres hacen de las citas.

\section{PROBLEMAS DE LAS DEFINICIONES Y APORTES DE LAS INVESTIGACIONES ${ }^{12}$}

\subsection{LiTERALIDAD DE UN DECIR O PENSAR AJENOS}

Uno de los problemas que se repite constantemente en las definiciones que ofrecen las gramáticas consultadas tiene que ver con la literalidad. Se afirma de forma reiterativa que el ED consiste en la reproducción literal de un decir o pensar ajenos. En la NGLE (p. 3275), por ejemplo, la definición destaca este aspecto y luego se matiza esta afirmación y se aclara que este procedimiento de cita "se caracteriza por la voluntad del hablante o del narrador de expresar literalmente la información que se transmite, de tal modo que la reproducción de las emisiones lingüísticas sea lo más exacta posible". Asimismo, se especifica que "esta literalidad del discurso directo

12 En este punto no me referiré a las variables sociales tomadas en cuenta en las investigaciones antes descritas (edad, sexo, grado de instrucción), pues no hay coincidencias que permitan ofrecer una conclusión generalizada en este sentido. 
reproducido es característica de las citas textuales y admite una gradación que va desde la transcripción exacta de la secuencia emitida [...] hasta traducciones o reelaboraciones más o menos aproximadas" (p. 3275). Se destaca, igualmente, que cuando se marca con comillas la información reproducida, esto tiene "consecuencias retóricas, puesto que induce en el lector cierto efecto de verosimilitud, a pesar de la inexactitud formal de lo que se transcribe" (p. 3276).

A mi juicio es más acertado acotar, como lo hacen Alcaráz Varó y Martínez Linares (2004: 240), que en el DD se trata, más bien, en el caso de la interacción oral, de una reproducción presentada como literal o tal como lo explica Portolés (2004: 218), quien también le atribuye literalidad al ED pero con más cautela: "El discurso directo se presenta, aunque casi siempre no lo sea, como una reproducción literal de las palabras propias o ajenas" ${ }^{13}$. Esta concepción se relaciona ampliamente con la idea de Mora y Álvarez 2003 sobre la iconidad y la verosimiltud que caracterizan las citas en estilo directo.

Otro aspecto que debería tomarse en cuenta es la posibilidad que tienen los hablantes, a propósito de la reflexividad del lenguaje, no solamente de citar lo que dicen o piensan los otros, pues también podemos referirnos a lo que hemos dicho o pensado nosotros mismos; acotación que se registra en la Nueva gramática básica (2011). Sobre este aspecto, también vale la pena destacar que esta reproducción de palabras puede desplazarse en el tiempo, es decir, no solamente podemos emplear las citas para reportar algo que ya se ha dicho, también podemos usarlas para poner en boca de alguien un enunciado que dirá a futuro:

(12) a. Ustedes dirán: "pero bueno, hay como una contradicción" (CARA_ M33_104)

b. La persona que no lo conozca dirá que él no habla mucho (CARA_ M13_082)

\subsection{ESTILO DIRECTO Y VERBOS DE REPORTE}

Otro aspecto que se debería destacar a la hora de definir el ED y el EI tiene que ver con la frecuencia de uso del ED en la oralidad, pues en todas las investigaciones descritas se observa la tendencia de los hablantes a usar en

13 Más información en este sentido en Reyes 1995 y 2002. 
sus conversaciones mucho más la cita directa que la indirecta, sobre todo si se trata de secuencias narrativas. Por tanto, tal como lo explica Benavent Payá 2003, la estructura marcada sería, sin lugar a dudas, el EI.

Asimismo, todos los verbos que se citan en la GDLE son muy poco productivos en la oralidad, pues el verbo usado por excelencia es decir, tal como se corrobora también en los estudios antes descritos.

La animacidad del sujeto citado también es un aspecto importante en el marco de las narraciones orales (cf. Cameron 1998). Por lo general, se trata de entes humanos animados, como en (13). Casos como los de (14) son muy poco frecuentes.

(13) a. Una enfermera me dice: "¿pero no tienes ninguno que te pueda comunicar a tu casa?” (CARA_H11_002)

b. Yo simplemente le dije que yo no quería estudiar más (CARA H11_003)

(14) Me acuerdo que el papel [...] decía más o menos lo siguiente: ahí te pongo $[\ldots]$ un carbón, un carbón porque te has portado muy mal en el año (CARA_H33_097)

Hay que acotar, sin embargo, que esto depende del contexto, específicamente del tipo de texto y de la situación comunicativa.

\subsection{Formas DE INTRODUCIR EL ESTILO DIRECTO E INDIRECTO}

Según lo que se desprende de las investigaciones comentadas, las citas en ED y EI, si bien es cierto que en su mayoría suelen introducirse mediante un verbo de reporte conjugado, como se describe en las gramáticas, también el ED puede introducirse por un verbo que no esté conjugado (15) y que no sea estrictamente de reporte (16).

(15) pues, uno decir: "tales días de la semana [...] los tengo libres" (CARA_H13_076)

(16) Cualquier vieja colombiana es: "¡mira vieja!, ¡mira viejo!” (CARA_ H13_075)

Igualmente, el marco introductor de la cita no debe ser necesariamente un verbo. Observamos las siguientes posibilidades de marco (Cameron 1998, Camargo 2004a, Gallucci 2009, Fernández 2011): 
(17) A partir de ahí yo estuve seis meses Ø: “sí, sí, cómo no, yo te hago las franelas como tú quieras" (CARA_H13_075)

(18) pasaba por una quebrada [...] Y QUE: "hasta mañana bachiller" (CARA_H33_097)

(19) Y Yo: “¡coño! pero esta mujer está loca vale” (CARA_H33_100)

(20) ENTONCES "No, abuelo. Quédese tranquilo, usted no se va a morir y tal" (CARA_H13_075)

En (17) tenemos una cita sin marco, o de ED libre, que es muy frecuente en la conversación y que no parece ser una variante del texto literario, como se afirma en el apartado 43.9d de la NGLE. Este uso parece estar relacionado con los pasajes dramáticos (van der Houwen 1998) y con la enumeración de actos de habla (García 2009). En (18) la cita se introduce mediante " $y+$ que"; en (19), con la conjunción y seguida de un sintagma nominal; en (20), a través de un marcador discursivo.

En cuanto al EI, es importante destacar que la conjunción que introduce la cláusula reportada no siempre es que, aunque se trata de la más usada en español. Al respecto, Verdín Díaz (1970: 52-53) afirma que no es necesario que la partícula indicadora de la subordinación sea una verdadera conjunción, como en el caso de El médico preguntó si María tenía fiebre, ya que si, al introducir una interrogativa indirecta, lejos de su valor de conjunción condicional, pasa a ser una partícula subordinante.

\subsection{Contenido de las Citas}

A propósito del contenido de las citas es importante llamar la atención sobre tres aspectos. En primer lugar, las citas no necesariamente deben estar constituidas sintácticamente por una cláusula reportada (Maldonado 1999). También pueden incluir fragmentos oracionales sin un verbo conjugado, como en (21).

(21) y yo le digo: “yo" (CARA_H11_002)

En segundo lugar, el contenido citado puede contener fragmentos que en realidad no citen palabras reportadas, como no se qué en (22), o puede estar constituido en su totalidad por estas estructuras que parecen usarse con la finalidad de llenar vacíos de información durante el intercambio comunicativo (23). Ambos casos constituyen una evidencia más a favor de la no literalidad de las citas directas en la oralidad: 
(22) Me decían: “mira, ven acá, haz esto, NO SÉ QUÉ” (CARA_H11_003)

(23) entonces [...] siempre me decían: “Y BROMA Y TAL" (CARA_H13_073)

En tercer lugar, hay que acotar, siguiendo a Cameron 1998 y a Camargo 2004a, que no solamente podemos citar palabras. También es posible reportar gestos, risas, onomatopeyas (24) e interjecciones (25).

(24) entonces a veces se la pasa jugando: “ija!” (CARA_M13_082)

(25) me encontré a la muchacha y me dijo: “¡hola!” (CARA_H13_074)

\section{REFLEXIÓN FINAL}

Como se ha visto hasta ahora, las definiciones que ofrecen las gramáticas sobre el estilo directo e indirecto son insuficientes, pues además de insistir en el carácter literal de las citas, especialmente en las de estilo directo, ilustran sus observaciones con ejemplos ideales o provenientes de textos escritos, sobre todo literarios, que distan mucho del funcionamiento real de estos procedimientos de cita en la interacción. Llama la atención, igualmente, que en la concepción del estilo directo e indirecto de Gili Gaya, Seco y Alarcos Llorach, por ejemplo, parece que solamente pueden citarse las palabras de los otros, y no las del propio hablante.

Sin duda alguna, a pesar de los problemas señalados, el capítulo de Maldonado 1999 en la Gramática descriptiva de la lengua española supone un avance importante -y una referencia obligada- en la explicación sintáctica del estilo directo e indirecto. No obstante, las definiciones deberían nutrirse de los resultados de investigaciones como las descritas y se debería proponer, por ejemplo, una categoría más estable de funciones discursivas del ED y el EI, sin olvidar la importancia del contexto en la construcción del significado, lo que nos lleva a subrayar la necesidad de un enfoque metapragmático (Reyes 1994, 1995, 2002) que dé cuenta del alcance real de las citas en la interacción oral.

\section{REFERENCIAS BIBLIOGRÁFICAS}

ACHARD, Michel. 1998. Representation of cognitive structures: Syntax and semantics of French sentential complements. Berlin: Mouton de Gruyter. 
Alarcos Llorach, Emilio. 1999. Gramática de la lengua española. Madrid: Espasa-Calpe.

Alcaraz Varó, EnriQue y María Antonia Martínez Linares. 2004. Diccionario de lingüística moderna. Barcelona: Ariel.

Alcina, Juan y José Manuel Blecua. 1975. Gramática española. Barcelona: Ariel.

Benavent PayÁ, Elisa. 2003. ¿Por qué contamos nuestras historias cotidianas en estilo directo? En Nicole Delbeque (ed.). Aproximaciones cognoscitivo-funcionales al español. Foro Hispánico 23: 11-20. Ámsterdam/ New York: Rodopi.

Bentivoglio, Paola e Irania Malaver. 2006. La lingüística de corpus en Venezuela: un nuevo proyecto. Lingua Americana 19: 37-46.

Bentivoglio, Paola y Mercedes Sedano. 1993. Investigación sociolingüística: sus métodos aplicados a una experiencia venezolana. Boletín de Lingüística 8: 3-35.

Briz Gómez, ANtonio (coord.). 1995. La conversación coloquial. Materiales para su estudio. Anejo XVI de la Revista Cuadernos de Filología. Valencia: Universitat de València.

CAMARGO, LAURA. 2004a. La representación del discurso en la narración oral conversacional. Estudio sociopragmático. Tesis doctoral, Universidad de Alcalá, Alcalá de Henares.

2004b. El estilo directo y el estilo indirecto. De las gramáticas del español al análisis del discurso y la pragmática. En Actas del V Congreso de Lingüistica General, vol. I, 479-489. Madrid: Arco/Libros.

2005. Cuando decir equivale a pensar. La función evaluadora de las citas directas en la conversación en español. Interlingüística 15: 237-246.

2008a. Cuestiones metodológicas de la investigación en pragmática: ¿de dónde proceden nuestros ejemplos? En José Antonio Calzón García (coord.). Actas del I Congreso Internacional de Filología Hispánica: Jóvenes Investigadores, pp. 81-92. Oviedo: Universidad de Oviedo.

2008b. La representación del discurso con los corpus PRESEEA: metodología y propuesta de análisis. Ponencia presentada en el XV Congreso Internacional de la ALFAL. Montevideo-Uruguay, 18-21 de agosto de 2008.

2008c. La metapragmática. En Ruiz Gurillo, Lorena y Xose Padilla (eds.). Dime cómo ironizas y te diré quién eres. Una aproximación pragmática a la ironía, pp. 88-108. Frankfurt: Peter Lang.

2010. Dialogues within oral narratives: Functions and forms. En Dale April and Lidia Rodríguez-Alfano (eds.). Dialogue in Spanish. Studies in functions and contexts, pp. 31-54. Amsterdam: John Benjamins.

- 2011. El discurso directo en el habla de Alcalá: ¿citan con fines distintos las mujeres y los hombres? Ponencia presentada en el XVI Congreso Internacional de la ALFAL. Alcalá de Henares-España, 6-9 de junio de 2011.

Cameron, Richard. 1998. A variable syntax of speech, gesture, and sound effect: Direct quotations in Spanish. Language Variation and Change 10: 43-83.

Coulmas, Florian. 1986. Direct and indirect speech. Berlin: Mouton de Gruyter.

Delbecque, Nicole. 2000. Cognitive constraints on complement clause cliticization in Spanish. En Kaoru Horie (ed.), Complementation, 149-197. Amsterdam: John Benjamins.

Fauconnier, Gilles. 1984. Espaces mentaux. Aspects de la construction du sens dans les langues naturelles. Paris: Minuit.

Fernández, María Fernanda. 2011. Uso del discurso directo e indirecto en el habla de Mérida. Tesis de grado para optar al título de Licenciada en Letras. Mérida: Universidad de Los Andes.

Gallucci, María José. 2009. Nos fuimos a la quebrada y mi mamá: “¿Estaban lanzándose por la quebrada?”, “¡No, mamá!”, “¡Claro que sí!”. Núcleo 26: 75-98. 
García, Renato. 2009. Aspectos pragmáticos y funcionales del discurso referido en la interacción cara a cara. Trabajo de grado para optar al título de Licenciado en Lingüística y Literatura Hispánica. Puebla: Benemérita Universidad Autónoma de Puebla.

García Mouton, Pilar y Francisco Moreno Fernández (dirs.). 2003. Atlas Lingüístico (y etnográfico) de Castilla-La Mancha. Alcalá de Henares: Universidad de Alcalá.

Gili Gaya, Samuel. 1998. Curso superior de sintaxis española. Barcelona: Bibliograf.

Gimate-Welsh, Adrián. 1985. Lenguaje, ideología y clases sociales. Puebla: Universidad Autónoma de Puebla, ICUAP, Centro de Ciencias del Lenguaje, Escuela de Filosofía y Letras, Colegio de Antropología.

Guirado, Krístel y Martha Shiro. 2004. Voces reportadas en las narraciones infantiles. Ponencia presentada en las XVII Jornadas Lingüisticas de la ALFAL, Universidad Central de Venezuela, Caracas.

Kerbrat-Orecchioni, Catherine. 1996. La conversation. Paris: Seuil.

Lázaro Carreter, Fernando. 1990. Diccionario de términos filológicos. Madrid: Gredos.

Lope Blanch, Juan Manuel (dir.). 1972. El habla de la ciudad de México. Materiales para su estudio. México: Centro de Lingüística Hispánica.

(dir.). 1976. El habla popular de la ciudad de México: materiales para su estudio. México: Centro de Lingüística Hispánica.

Maldonado, Concepción. 1991. Discurso directo y discurso indirecto. Madrid: Taurus. 1999. Discurso directo y discurso indirecto. En Ignacio Bosque y Violeta Demonte (eds.). Gramática descriptiva de la lengua española, Vol. 3: 3551-3595. Madrid: Espasa Calpe.

Mateus, Ligia. 2005. El estilo directo e indirecto como estrategias narrativas en el habla de Caracas. Trabajo de grado para optar al título de Licenciada en Letras, Universidad Central de Venezuela, Caracas.

Mora, Elsa y Alexandra Álvarez. 2003. Indirect discourse and its acoustic/prosodic characteristics in Venezuelan Spanish. En María Josep Solé, Daniel Recasens y Joaquim Romero (eds.). Proceedings of the 15th International Congress of Phonetic Sciences, pp. 571-574. Barcelona: Causal Productions.

Portolés, José. 2004. Pragmática para hispanistas. Madrid: Síntesis.

Real Academia Española. 1973. Esbozo de una nueva gramática de la lengua española. Madrid: Espasa Calpe.

2009. Nueva gramática de la lengua española. Tomo II. Madrid: Espasa.

2010. Nueva gramática de la lengua española. Manual. Madrid: Espasa.

2011. Nueva gramática básica de la lengua española. Barcelona: Espasa.

Reyes, Graciela. 1994. La pragmática lingüística. El estudio del uso del lenguaje. Barcelona: Montesinos.

1995. Los procedimientos de cita: estilo directo y estilo indirecto. Madrid: Arco/Libros.

2002. Metapragmática. Lenguaje sobre lenguaje, ficciones, figuras. Valladolid: Universidad de Valladolid.

San Martín, Abelardo y Silvana Guerrero. 2012. Discurso referido y oralidad en el corpus PRESEEA de Santiago de Chile. Ponencia presentada en el Coloquio Interacción y manifestaciones discursivas en el diálogo oral. Santiago de Chile, 3-4 de octubre de 2011.

Seco, Manuel. 1998. Gramática esencial de la lengua española. Madrid: Espasa Calpe.

Thompson, Geoff. 1994. Reporting. London: HarperCollins Publishers.

van DER Houwen, Fleur. 1998. Organizing discourse. Direct and Indirect Speech in Mexican Spanish. Linguistics in the Netherlands 15: 123-134. 
2000. El habla directa vs. indirecta y la organización del discurso. Foro Hispánico 17: $27-40$.

Verdín Díaz, Guillermo. 1970. Introducción al estilo indirecto libre en español. Revista de Filología Española, Anejo CXI. Madrid: CSIC. 